\title{
Magnetic braking and ambipolar diffusion in metal-poor protostars
}

\author{
Hans Zinnecker \\ Astrophysikalisches Institut Potsdam, An der Sternwarte 16, D-14482 Potsdam, Germany \\ email: hzinnecker@aip.de
}

\begin{abstract}
In this short note, we raise the issue how magnetic braking of prestellar and protostellar condensations depends on the metallicity of the molecular gas cloud. We suggest that the degree of ionization, which determines the timescale for redistribution of magnetic flux (ambipolar diffusion) in the dense cloud core, depends linearly on the heavy element and dust abundance of the gas. This implies that magnetic braking is less efficient in metal-poor condensations, and hence more of the angular momentum problem of star formation must be solved by other means, such as fragmentation into (wider) binary systems. Observations of orbital periods (separations) of binary systems among the metal-poor Galactic halo stars could test this prediction.
\end{abstract}

Keywords. Magnetic fields - diffusion - protostars - ISM: molecules

\section{Angular momentum problem in star formation}

Magnetic braking of rotating, slowly contracting, self-gravitating protostellar cores via torsional Alfven-waves to the outside cloud medium can help to solve the angular momentum problem in low-mass star formation (Ebert et al. 1960, Mestel 1965, Mouschovias and Paleologou 1979). In the case of an aligned magnetic rotator up to two orders of magnitude in specific angular momentum $(J / M)$ can be lost by magnetic braking, before dynamical collapse sets in due magnetic flux redistribution (ambipolar diffusion) in the core, at central gas densities $>10^{4}$ to $10^{6} \mathrm{~cm}^{-3}$ ). We refer to the key papers of Basu and Mouschovias (1994, 1995ab) and the review by Mouschovias (1996).

\section{Ambipolar diffusion}

The timescale for ambipolar diffusion to drive the core unstable is (Mouschovias 1996):

$$
t(A D)=2\left(x_{i} / 10^{-7}\right) M y r
$$

where $x_{i}$ is the fractional degree of ionisation $\left(x_{i}=n_{i} / n_{\text {tot }}\right)$ which scales as $n_{t o t}^{-1 / 2}$ for total molecular gas density $n_{\text {tot }}$ (Elmegreen 1979, Nakano 1979). The scaling constant is proportional to the metal abundance and the dust content of the molecular gas (with Fe, $\mathrm{Mg}$, Na being the dominant donors of free electrons and most grains negatively charged). Cosmic rays (100 MeV protons) provide a steady source of ionizing flux (Elmegreen 1979).

\section{Observational prediction for metal-poor stars}

The theoretical prediction is that the ambipolar diffusion timescale is shorter for lowermetallicity molecular gas (owing to the lower fractional degree of ionisation $x_{i}$ ). Thus the collapse of protostellar cores in metal-poor conditions (such as in the LMC/SMC and in the early Galactic halo) would result in higher angular momentum systems, i.e. wider 
binaries and/or faster rotating stars. This prediction can be tested observationally (cf. Mouschovias 1977; Zinnecker et al. 2004).

\section{Magnetic collapse: from subcritical to supercritical cores}

Strong magnetic fields inhibit molecular cloud collapse and star formation. There is a critical mass which must be exceeded for a given magnetic field strength $B$ and cloud gas density $n$

$$
M(\text { crit })=5(B / 10 \mu G)^{3} /\left(n / 10^{4} \mathrm{~cm}^{-3}\right)^{2} M_{\odot}
$$

for gravitational instability to begin. We call a molecular cloud core "supercritical" when the magnetic field is not strong enough to prevent collapse, "subcritical" when it can prevent collapse. Even in the latter case, a molecular cloud core evolves through quasi-static contraction through the motion or slow drift of neutral gas past the ionized component (ambipolar diffusion). It is only the ionized component (electrons, charged grains) which is held up by magnetic forces while the neutral gas is coupled to the ionized component by frequent ion-neutral collisions. If this coupling is perfect, the magnetic flux is frozen into the cloud. At high densities (typically in excess of $10^{6} \mathrm{~cm}^{-3}$ ) the degree of ionization in cloud cores starts to drop sufficiently (from $10^{-6}$ to $10^{-8}$, say) so that the assumption of flux freezing breaks down. Then ambipolar diffusion starts to redistribute the magnetic flux $\Phi=B R^{2}$ in the center of the cloud core, thereby increasing the central mass-to-flux ratio until it exceeds the critical value for collapse: the core goes "supercritical". An inside-out dynamical collapse (somewhat retarded compared to free-fall without magnetic forces) sets in; the prestellar core turns into a real protostar, including a magnetized rotating disk and bipolar jet!

3D MHD simulations of collapsing rotating magnetic $1 M_{\odot}$ cloud cores, using adaptive mesh refinement techniques, have been published (Ziegler 2005) which show that these cores can fragment into wide binary systems, when the core mass-to-flux ratio exceeds the value for supercritical collapse by a factor of about 2, even if the magnetic flux is frozen (i.e. without ambipolar diffusion).

\section{Acknowledgements}

The author thanks John Beckman and Klaus Strassmeier for making my attendance possible. It is a pleasure to thank T. Mouschovias and U. Ziegler for extensive discussions.

\section{References}

Basu, Sh. \& Mouschovias T.Ch. 1994, ApJ 432, 720

Basu, Sh. \& Mouschovias T.Ch. 1995a, ApJ 452, 386

Basu, Sh. \& Mouschovias T.Ch. 1995b, ApJ 453, 271

Ebert, R., v. Hoerner S., \& Temesvary, St. 1960, in: Die Entstehung von Sternen durch Kondensation diffuser Materie, Springer Verlag, pp. 290

Elmegreen, B. G. 1979, ApJ 232, 729

Mestel, L. 1965, Quarterly Journal of the Royal Astronomical Society 6, 265

Mouschovias, T.Ch. 1977, ApJ 211, 147

Mouschovias, T.Ch. \& Paleologou E. V. 1979, ApJ 230, 204

Mouschovias, T.Ch. 1985, A\&\&A 142, 41

Mouschovias, T.Ch. 1996, in: Solar and Astrophysical Magnetohydrodynamic Flows, eds. K. C. Tsinganos, Kluwer Academic Publishers, p. 505

Nakano, T. 1979, PASJ 31, 697

Ziegler, U. 2005, A\&A 435, 385

Zinnecker, H., Köhler R., \& Jahreiß, H. 2004, RevMexAA 21, 33 